\title{
The Evolution of Physiotherapy in Animal Rehabilitation in Durban, Kwazulu Natal
}

\begin{abstract}
Introduction: A nimal physiotherapy is a specialized branch of physiotherapy. As this specialization is not introduced at undergraduate level, there is a need to ascertain whether qualified physiotherapists had an interest in this field. The need for post-graduate interest in this subject would support a post-graduate programme as there is no academic institutional programme in this field in South Africa.

Aim of study: To gain information on the awareness of and interest in animal physiotherapy from veterinary surgeons and qualified physiotherapists.

Methodology: Two questionnaires were constructed, one for veterinary surgeons and one for physiotherapists. Sixty physiotherapists and 30 veterinary surgeons in private practice in Durban, KwaZulu Natal participated in the study. A return of $73 \%$ of the questionnaires for veterinary surgeons and 65\% for physiotherapists was obtained.

Data analysis: Data for Physiotherapists and veterinary surgeons were grouped together for responses to the questionnaire and compared.

Results: All physiotherapists and veterinary surgeons involved in the study are aware of animal physiotherapy. $81.5 \%$ of the veterinary surgeons administer physiotherapy treatment as part of their overall rehabilitation programme and $7.41 \%$ refer their clients to physiotherapists. All the veterinary surgeons stated that physiotherapy is necessary in the overall animal rehabilitation. $12.5 \%$ of physiotherapists treated animals and all felt under-qualified to treat animals. Conclusion: The study reveals a need for post-graduate specialization in animal physiotherapy and this would promote public awareness of its existence and benefit to animals.
\end{abstract}

KEYWORDS : ANIMAL PHY SIOTHERAPY, VETERINARY PRACTICE.

\section{INTRODUCTION}

\subsection{Background}

Prior to World War II diversification into animal physiotherapy was pioneered by Sir Charles Strong (Wihelm, 2003). Animal physiotherapy began earning recognition in the 1980's when the Association of Charted Physiotherapists in Animal Therapy (ACPAT) was founded.

\section{Correspondence to:}

Nirmala Naidoo

Discipline of Physiotherapy

School of Physiotherapy,

Sports Science and Optometry

Faculty of Health Science

University of KwaZulu-Natal

Private Bag X54001

Durban 4000 South Africa

Tel: +27312607817

Fax: +27312608106

e-mail: naidooni@ukzn.ac.za
It was at this time that the field rapidly began gaining ground, becoming more understood, studied and accepted (Boyle, 2002). The combination between human and natural sciences was the unique characteristic of human-animal interaction as a field of study. This combination led to educators (in the fields of physiotherapy and the veterinary sciences) offering joint discipline courses to both physiotherapy and veterinary students in the United Kingdom and Australia (Levine, 1997).

Over recent years, physiotherapists have been incorporating the basic principles of physiotherapy into animal rehabilitation (personal experience). Thus it has become the practice of some veterinarians interested in optimizing the rehabilitation of their patients, to understand the benefits of physiotherapy and to collaborate with a physiotherapist to provide holistic care to their clients (Wihelm, 2003).
1.2 International curriculum for animal physiotherapy

The field of animal physiotherapy is swiftly progressing worldwide. The literature informs that internationally the scope for animal physiotherapy is broadening and advancements in treatments are being made. Countries internationally involved in research and development in animal physiotherapy include Australia, the United Kingdom, Canada and the United States of America. Internationally, the qualifications required before practicing animal physiotherapy is a degree in Physiotherapy, registration with the Licensure Borad of the country where practicing, completion of at least 2 years of general practice working in the human field and attendance of specialist animal physiotherapy courses. Course curriculum includes core knowledge, and skills course and clinical placements. These are designed to give the 
student a sound basic practical and theoretical grounding in animal physiotherapy. Students are examined in both practical and written components. In Australia, the University of Quuensland offers a Masters programme and can be accessed via distance learning. Professional liability is required to treat animals.

In South Africa, the physiotherapy curriculum does not include the teaching of animal rehabilitation. Practicing physiotherapists, whether treating humans, animals, or both, are required to register with the Health Professions Council of South Africa (HPCSA). The special interest group, the Animal Physiotherapy Group of South Africa (APGSA), is a sub-group of the South African Society of Physiotherapy. In June 1996, due to the establishment of the APGSA, the legal practice of animal physiotherapy in South Africa was accepted by the South African Society of Physiotherapy (SASP).

It was therefore decided to investigate the interest in animal physiotherapy amongst qualified Physiotherapists as well as the veterinarian surgeons' perceptions of animal physiotherapy in Durban, KwaZulu Natal.

\subsection{Purpose of study}

The study is intended to establish the involvement of qualified physiotherapists in the practice of animal physiotherapy and to establish the extent of the veterinarian surgeons' knowledge of animal physiotherapy.

\subsection{Significance}

The significance of the study is to determine the number of physiotherapists currently involved in animal rehabilitation in Durban, KwaZulu Natal (KZN) as well as whether the demand, if any, for animal physiotherapy is being met. It is also to establish the awareness and attitude of veterinary practitioners toward animal physiotherapy and its perceived results in animal rehabilitation. The results obtained will assist in determining the need for specialist animal physiotherapists in Durban, KZN as well as the possible impact on undergraduate and post-graduate physiotherapy curriculum at our physiotherapy training institutions.

\section{LITERATURE REVIEW}

A review of the databases Pubmed, MEDLINE and the Cochrane library reveal that animal physiotherapy is increasing in popularity internationally, however there is no formal education structure for animal physiotherapy within South Africa. No study has been undertaken in Durban, KZN investigating the awareness of animal physiotherapy among veterinary practitioners. One similar study was undertaken in the United Kingdom, investigating veterinary practitioner's perceptions of animal physiotherapy (Knowles and Mackintosh, 1994).

\subsection{Animals most commonly treated}

Animals most commonly treated by physiotherapists include horses, dogs, cats and birds (Mackintosh and McNamara, 1993). Occasionally physiotherapists may treat lions, cheetas, snakes, baby rhino, rabbits, sheep, goats, cattle and elephants (Dunlop et al, 1970; Mackintosh et al, 1994). Horses and dogs are the most commonly treated animals by both physiotherapists and veterinary surgeons. This can be attributed to their breeding for performance and competition where speedy and immediate rehabilitation is essential to return the animal to their peak athletic ability (Bromiley, 1989). Owners as well as breeders are therefore encouraged to consult with animal physiotherapists. Research has shown that popularity toward animal physiotherapy increases when injury ensues in livestock of a higher value (Knowless and Mackintosh, 1994).

\subsection{Commonly treated injuries in animals}

Common conditions treated in animals by both physiotherapists and veterinary surgeons were reported by Knowless and Mackintosh (1994). They reported that as with humans, conformation (physique) and lack of fitness are two of the predominant factors causing injury in animals. Behaviour patterns, the result of captivity, rider errors in horses and owner errors in dogs (performance tasks animal not designed to perform) commonly result in injury. The authors found that the most common conditions include the following in specific animals:
Horses: Painful necks and backs followed by tendon and ligament damage, wound abscess, skin disease, muscular problems i.e. tears and atrophy conditions, contractures, conditions such as splints, painful shins, sand crack (slit in the outer wall of the hoof involving the coronary band), capsulitis as a result of sprains, bruising as a result of direct trauma, bursitis, degenerative arthritis, stifle lameness and fractures of the pastern are the commonest conditions treated by an animal physiotherapist (Bromiley, 1989).

Dogs: Tendon and ligament trauma especially cruciate ligament repair, back problems, osteoarthritis, hip dysplasia, muscular problem, wounds, skin conditions, rheumatic conditions, disc and soft tissue lesions (Steiss, 2002).

Other animals: Physiotherapy is recommended in treatment of stifle ligament in sheep and cattle, back problems in sheep, goats, cats, cattle, musculoskeletal problems in cows, calves, sheep and cats, tendonitis in bulls, meningitis in rabbits, pneumonia in snakes as well as following wing repair in birds (Knowless and Mackintosh, 1994).

\subsection{Assessment and diagnosis of animals}

Assessment commences with a veterinary referral form and includes the subjective and objective assessment (Steiss, 2002). A physiotherapist requires information pertaining to the breed and occupation of the animal, diagnosis, present medical history, tests and investigations, past medical history, medication as well as surgical history before commencing with the subjective component. Assessing and diagnosis of animal conditions is often very challenging but greater powers of observation and palpation soon develop (Steiss, 2002).

The subjective assessment includes, but is not limited to, the following:

- Description of dogs main problem

- Determination of insidious or traumatic onset and constant or intermittent affliction

- Changes observed in: bladder or bowel function; sitting or lying position; tail carriage; appetite and diet

- If chewing of the fore-or hind limbs has been observed 
- If the animal is able to lie on its back or with its legs out behind it

- Where and what the animal sleeps on

- Description of the animals usual and current level of physical activity and the terrain

- If the animal wears a check-chain or harness

- Description of the animals swimming style

The objective examination includes the following:

- Static assessment of posture cranially, caudally and laterally

- Videotaped gait analysis at slow, medium and fast paces

- Tight circling ability to left and right

- Condition of hooves, pads and claws

- Thoracic sling mobility and muscle condition

- Sit and lying position analysis

- Sit to stand and sit to lying analysis

- Joint range of motion

- Muscle bulk

- Ligament tests

- Surface palpation findings.

\subsection{Treatment modalities}

A treatment programme and home programme follows the one and a half-hour assessment (Bromiley, 1993). Treatment methods concerning animal physiotherapy vary greatly. Many are adapted from physiotherapy to humans and many have been devised especially for animal use. As in human physiotherapy, new methods of treatment are continually being developed. Modalities used are much the same as in humans, however dosage, duration and application may differ (Mills and Levin, 1997). The following are some of the modalities used in the physiotherapy treatment of animals:

Massage: Massage may be defined as the systemic and scientific manipulation of soft tissue usually for therapeutic use (Mills et al, 1997; Lawson et al, 1970). Massage of injured tissue is commonly employed by animal trainers for relaxation of soft tissue, decrease muscle spasm, desensitize trigger points, increase muscle flexibility, improve venous and lymphatic flow with reduction of oedema and increase local blood flow to the area. Equine sports massage is the therapeutic appli- cation of professional sports massage techniques to the horse. These hands on techniques accomplish increased performance allowing the horse to compete to its full potential and increase endurance levels (Bromiley, 1993). The value of massage oils is controversial, but the massage itself is considered extremely beneficial (Bromiley, 1989). Although massage is a physiotherapeutic modality, commercial products such as massage pads, strapping pads and body brushes illicit the desired effect and can be administered by animal owners thereby limiting physiotherapy consults.

Superficial heat modalities: Bromiley (1993) advocated superficial heating agents to heat the skin and subcutaneous tissues to a depth of two centimeters. Deeper tissues may be heated through conduction especially with long term application. Heating agents include hot packs, radiant heat, ultraviolet light, infrared, solarium, shortwave diathermy, heating blankets and warm baths. The application of heat is beneficial for its analgesic and increase circulatory effects. Heat promotes local vasodilatation at temperatures up to $42^{\circ} \mathrm{C}$. It reduces pain by inducing a sedative effect on sensory nerve endings, increase metabolic activity in skin and underlying superficial tissue, relief of joint stiffness following injury, general relaxation, decrease muscle spasm, promotes tissue extensibility of joint capsules, tendon and scar tissue (Lawson, 1970; Mills and Levine, 1997).

Cryotherapy: The application of cold decreases blood flow locally through vasoconstriction and this helps to reduce the effects of inflammation and hemorrhage and decrease metabolic rate of the local tissues. Application includes ice massage, water boots (where the limb is emerged in container filled with crushed ice), aqua boots, frozen gamgee, bonner bandages, frozen cotton wool and non-toxic cooling gels. Treatment time is approximately twenty minutes before vasodilatation occurs (Hopes, 1970).

Hydrotherapy: In veterinary terms, hydrotherapy is taken to mean the treatment of disease pathology in water (Mills and Levine, 1997). Swimming is a form of rehabilitation particularly appropriate for dogs and horses recover- ing from a range of conditions especially those involving problems with the limbs. As all animals swim by instinct, swimming presents as an ideal exercise which helps improve muscle tone and is helpful when recovering from injury or operation. Muscle weakness generally follows immobilization. In order to prevent further weakness or injury, it is important to rebuild wasted and weak muscles using safe exercises. Swimming effectively encourages all limbs to stay afloat thereby exercising all joints and muscles without the impact of hard ground on weight-bearing on the joints. Many race horse and dog trainers use hydrotherapy as part of the regular fitness regime (Bromiley, 1993). Heated water is best used when dogs are swimming since cold water causes constriction of superficial blood vessels which restricts the flow of blood making superficial muscles less efficient. Cold water is however used for horses as it helps dissipate the enormous amounts of heat they generate under exertion. Swimming improves general fitness of the animal by enhancement of the cardiorespiratory system. Hydrotherapy is beneficial in strengthening muscles, but does very little for bone and must always be used in conjunction with exercises on land (Mills and Levine, 1997).

Other: Other treatment modalities include passive movements, acupuncture, modified Frenkel's exercises, ultrasound, interferential and laser therapy.

\section{METHODOLOGY}

\subsection{Subjects}

Physiotherapists in both private and public service practicing full-time in the central Durban area and veterinary surgeons in Durban, KwaZulu Natal area were included in the study. Stratified random sampling was used.

\subsection{Instrumentation and Procedures}

Two separate questionnaires were used. One questionnaire was specific to the physiotherapists and the other to the veterinary practitioners. The questionnaire was designed to be concise and consisted of both open and closed-ended questions. A pilot study was initially undertaken to test the content validity of the questionnaire. The questionnaires were hand delivered to the subjects 
and collected on completion. Subjects were given one week to complete the questionnaire. Personal administration of questionnaires was to establish a rapport with the sample groups to explain the purpose of the study. The same researchers who distributed the questionnaires collected them on completion. The results of the pilot study were included in the final results.

\subsection{DATA ANALYSIS}

The data was divided into sub groups according to responses to specific questions, practicing and non-practicing animal physiotherapists and referring and non-referring veterinary surgeons. The responses by both groups of subjects (physiotherapists and veterinary surgeons) were also compared. Statistical functions in the Microsoft Excel programme were used to generate analysis of the data.

\section{RESULTS}

Sixty questionnaires were received from physiotherapists and thirty from the veterinary surgeons $(100 \%$ effective response rate). The questionnaires were divided according to those physiotherapists who had treated animals and those who had not treated animals and grouped accordingly. The results were divided and analysed in three main sections as follows: separate analyses for each group, namely, the physiotherapists and the veterinary surgeons and a comparative analysis of both groups.

\section{Results obtained from questions specific to Physiotherapists}

Of the 60 physiotherapists involved in our study, 48 were employed in private practice. Only 6 of this cohort had treated animals and none were qualified in animal physiotherapy.

Figure 1 illustrates the physiotherapists' views on animal physiotherapy. None were adequately qualified; all agreed on the need for animal physiotherapists and $16.7 \%$ were aware of animal physiotherapy courses. $97.9 \%$ of the study cohort of physiotherapists was accepting of this field physiotherapy as being specialized.

Physiotherapists reported that the most common modalities used were heat, ultrasound, acupuncture, laser,

Figure 1: Graph indicating the views of physiotherapists' on animal physiotherapy

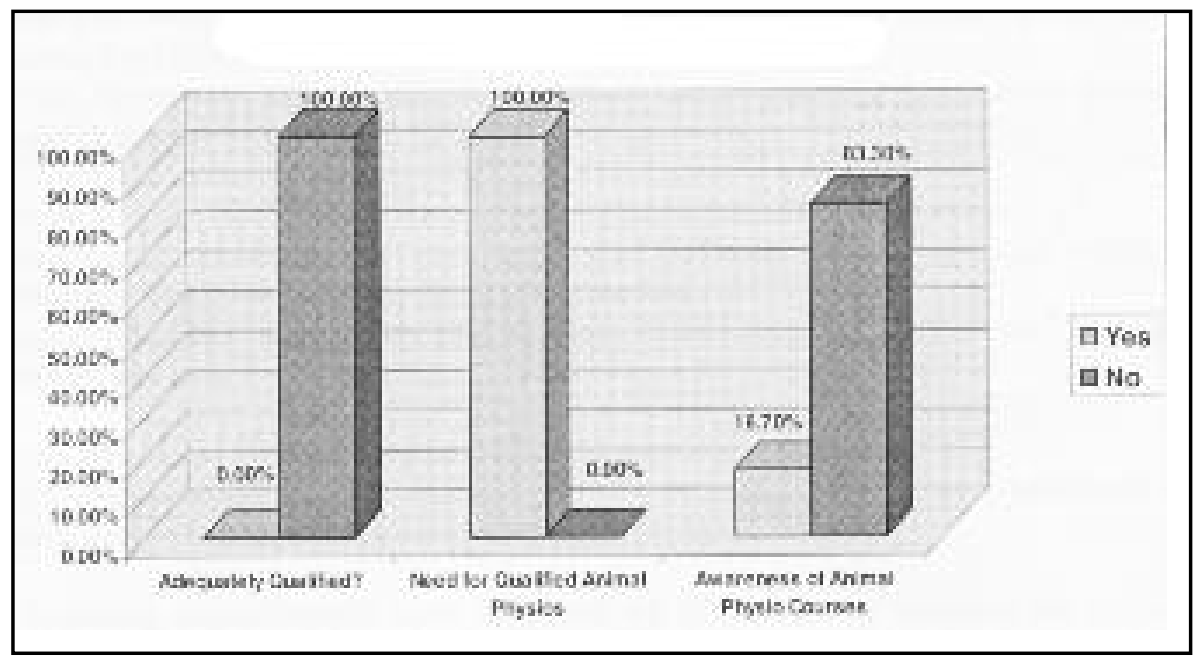

hydrotherapy and sea sand, which was in keeping with the study done by Bromiley (1993). As found in this study, animals most commonly treated were dogs, horses and cats.

The results obtained regarding undergraduate introduction to animal physiotherapy. 58.54\% of Physiotherapists agreed on an introductory course being implemented at undergraduate level. A minority of physiotherapists $(12.5 \%)$ of the study cohort practice animal physiotherapy. $100 \%$ of the physiotherapists in the study did not feel adequately qualified to administer physiotherapy to animals.

Injuries in animals commonly treated by physiotherapists included the following:

Osteoarthritis, laminectomy, ankylosing spondylitis, spondylosis, post spinal decompression, musculoskeletal conditions. Physiotherapists who practice animal physiotherapy reported that they acquired their patients as follows:

Own pets, exercises given to pet owner by physiotherapist or the veterinary surgeon and consultation with the physiotherapist by veterinary surgeon.

\section{Results obtained from questions specific to the veterinary surgeons}

$7.4 \%$ of all veterinary surgeons' responses revealed that they refer their clients to physiotherapists.

In contrast to the previous question, $81.3 \%$ of the veterinary surgeons in this study adopted their own Physiotherapy treatment for their clients. They listed the following modalities used as follows:

Heat, ice, ultrasound, acupuncture, hydrotherapy, action potential stimulation, passive movements and infrared.

The veterinarians also listed the most commonly treated conditions as follows:

Musculoskeletal, post-operative care, spinal disc pathology, spinal pathology arthritis, inflammatory conditions and neuromuscular disorders.

Animals commonly treated are horses, dogs (Giant breeds, Dachshunds, German Shepherds) and cats.

Veterinarians found the following limitations to animal physiotherapy: Physical animal handling, unco-operative patients (animals), animal owner adherence to treatment regimen, time (assessment and treatment time).

The following improvements were suggested by the veterinary surgeons for animal physiotherapy:

Training and implementation, public awareness, increase research in animal physiotherapy, close liaison with veterinary surgeons, facility incorporating hydrotherapy, acupuncture and massage, improve veterinary surgeons' knowledge, post-graduate degree/ diploma in animal physiotherapy and integration of physiotherapists into veterinary clinic.

$100 \%$ of the veterinary surgeons stressed the need for animal physiotherapy in animal rehabilitation.

Comparison of Physiotherapists' and Veterinary surgeons' responses

The awareness of animal physiotherapy was $97.9 \%$ for physiotherapists and $100 \%$ for veterinary surgeons. 
Figure 2 illustrates comparatively the source of awareness of physiotherapists and veterinary surgeons about animal physiotherapy. Articles written about animal physiotherapy (SASP journals, magazines and newspapers) served to be the most common resource amongst physiotherapists at $60.4 \%$, and colleagues served to be the most common amongst veterinarians at $59.2 \% .14 .6 \%$ of physiotherapists had learnt about animal physiotherapy in their undergraduate training.

$20.8 \%$ of physiotherapists and $82.6 \%$ of veterinarians expressed willingness to include animal physiotherapy in their practice.

When comparing the interest to learn more about animal physiotherapy, it was noted that veterinarians showed a higher interest at $92.6 \%$ as opposed to the $65.9 \%$ of physiotherapists who are willing to learn more about animal physiotherapy. However, as illustrated in Figure 3, 91.9\% of physiotherapists and $77.8 \%$ of veterinary surgeons expressed the need for physiotherapists to specialize in animal physiotherapy as a postgraduate qualification.

\section{DISCUSSION}

Physiotherapy has been recognized as a fast growing field internationally. The establishment of postgraduate academic programmes in animal physiotherapy gives physiotherapists accessibility to specialization in this field. However, specialist qualifications in this field are not offered within South Africa and physiotherapists wishing to pursue this field have no access to specific training. It is evident by the results obtained from this study that there is a need for animal physiotherapy specialization and this field could be lost to other allied professions if neglected by the physiotherapy profession in South Africa.

Animal physiotherapy is gaining ground locally especially with the development and growth of the APGSA, subgroup of the SASP. However, a physiotherapist wishing to specialize in animal physiotherapy is required to study internationally, making specialization both difficult and expensive. Additionally, as the majority of physiotherapists displayed an interest in this field and reported that they were

Figure 2: Graph illustrating the comparison of responses relating to the awareness of animal physiotherapy.

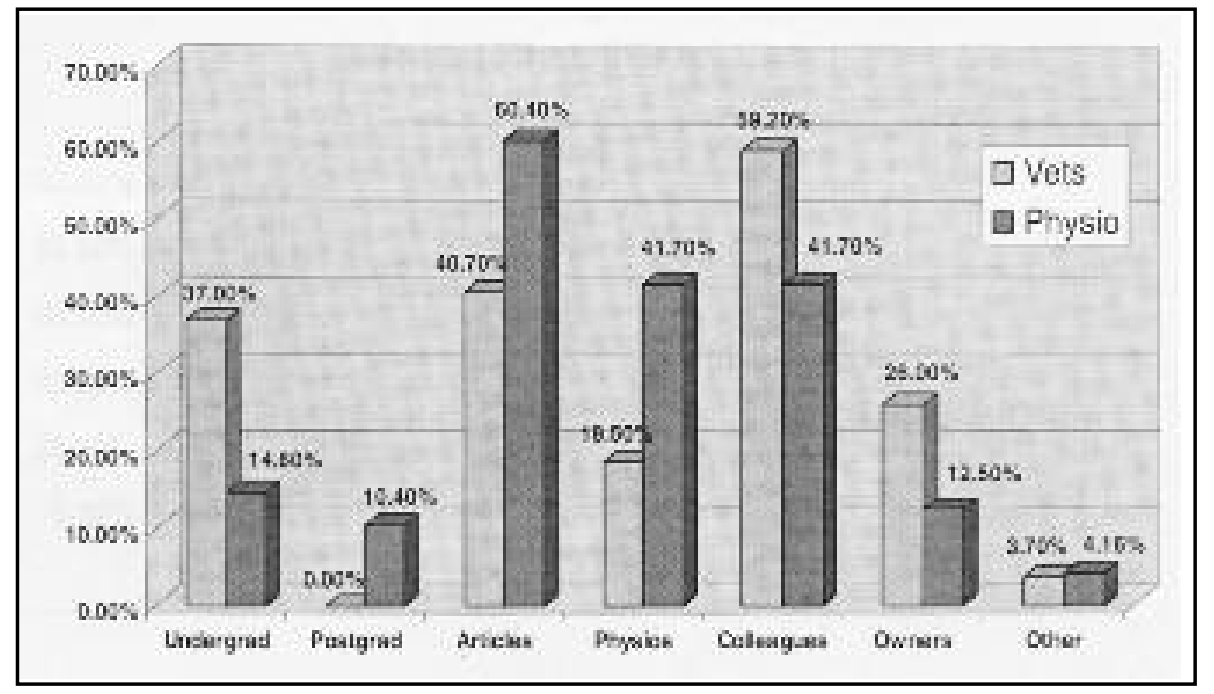

unaware of any local courses that are conducted, clearly indicates a need for local courses to be developed and conducted for interested physiotherapists.

Presently, all cases must be treated on referral from a veterinary surgeon. Although physiotherapists (a minority, as shown in the results) are practicing animal physiotherapy, there was no report of a rehabilitation team involved with animal care. The physiotherapists and veterinary surgeons report working in general isolation of each others practice. This is in keeping with the study by done by Steiss (2002) and may be attributed to lack of qualification in this field (and possible lack of confidence to practice in a team).

A minority of veterinary surgeons' responses revealed that they refer their clients to physiotherapists. The majority administer physiotherapeutic treatments themselves. This may be due to the paucity of physiotherapists who do practice animal physiotherapy and also the lack of speciality in animal physiotherapy. Therefore, there is a clear need for physiotherapists to explore the field of animal physiotherapy as a new frontier.

Physiotherapists in our study displayed interest to learn more about animal physiotherapy. This can be interpreted as an interest in this field. The main source of awareness was attributed to articles being published by APGSA in the SASP journals, which further illustrates interest in this field. There is thus necessity to develop and stimulate this interest amongst physiotherapists if we are to enhance our profession in this field.

Physiotherapists believe that animal owners are unaware of the important role animal physiotherapists play in holistic animal treatment. The results show that the number of practicing physiotherapists in the field of animal physiotherapy is sparse. Although physiotherapists feel that there is poor public awareness, there is also lack of awareness about animal physiotherapists among practicing physiotherapists themselves. There is keen awareness and interest among physiotherapists to learn more about this field of specialization (Figure 3), however a lack of formal qualification on the part of physiotherapy may be viewed as one of the inhibitions to further the interest of this specialization. As there are no animal physiotherapy post-graduate courses offered in South Africa, this may provide motivation for the design and implementation of training in this area of physiotherapy.

Veterinarians expressed their willingness to work in conjunction with animal physiotherapists and share practice with them. This is in line with the results obtained in a study done by Mackintosh and McNamara (1993). The authors reveal that large animal owners would be more willing to pay a greater holistic treatment fee for animals that were of greater value or an asset to the owner. Examples are performance animals and those bred as livestock for sale. This is further incentive and motivation for the 
Figure 3: Comparison of veterinarians' and physiotherapists' views on postgraduate specialization in animal physiotherapy

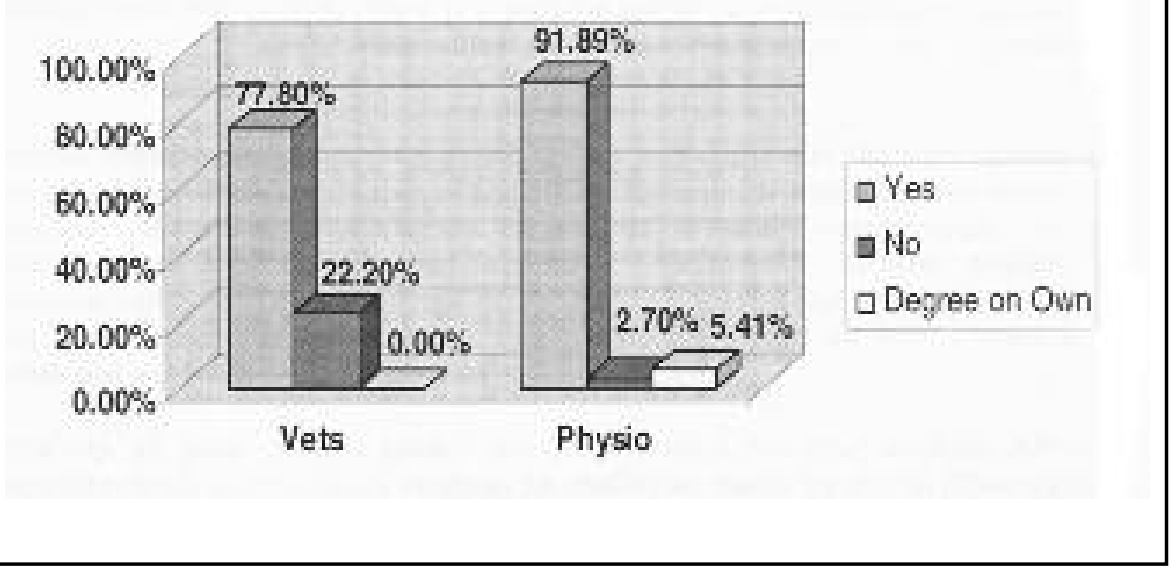

need for this specialist field in South Africa. This is verified by the studies done by Bromiley (1989) and Knowless and Mackintosh (1994).

\section{LIMITATIONS}

The study was limited to a cohort of physiotherapists and veterinary surgeons within the central Durban area of KwaZulu Natal. It is known from personal communication that there are practicing animal physiotherapists outside the central business district of Durban (CBD). The area outside the CBD would provide for more space and opportunity for the practice of animal physiotherapy as many stables, etc are located outside the CBD (refer Recommendations).

\section{CONCLUSION}

There is awareness amongst both physiotherapists and veterinary surgeons in the field of animal physiotherapy, but it is agreed that a lack of qualification on the part of the physiotherapists is hindering its progression as a specialist field. Both groups of practitioners involved in the study felt that postgraduate specialization is necessary for the further development of the profession. A definite agreement on its benefits in holistic animal rehabilitation was emphasized by both practitioners. Veterinarians displayed positive responses in favour of physiotherapists becoming specialized in animal physiotherapy, as well as emphasizing its need in holistic animal rehabilitation. Close consultation between veterinarians and animal physiotherapists is essential to develop a professional rapport and construct a referral system to optimize the animal physiotherapy service.

Interest amongst physiotherapists to learn more in the field of animal physiotherapy exists, but without advertising and creating public awareness, animal physiotherapy is not going to meet its needs. Nationally conducted studies are mandatory to establish the need and progression for animal physiotherapy. Further research and availability of courses for specialization are necessary to prevent this field of physiotherapy being lost to outside professions, if not lost altogether.

\section{RECOMMENDATIONS}

The following recommendations are suggested as a sequel to this study:

- There needs to be a public awareness drive to create public awareness on the existence and benefit of animal physiotherapy. This should be concurrent with the promotion of animal physiotherapy to veterinary surgeons and physiotherapists.

- A national study amongst veterinarians and physiotherapists will help determine the need and feasibility for a postgraduate academic programme in animal physiotherapy in South Africa. This would also provide a more realistic figure on the number of practicing animal physiotherapists.

- Another study to investigate the inclusion of aspects of animal physio- therapy in the undergraduate physiotherapy curriculum would provide background to this specialist field and stimulate further interest for postgraduate study.

- Liaison with the veterinary schools in South Africa to foster and enhance collaboration for the development of post-graduate courses and qualification in this field of specialization within physiotherapy.

\section{REFERENCES}

Boyle, K (2002) Physiotherapy in Veterinary Medicine. Physiotherapy, 8, 9, 14.

Bromiley, M (1993) Equine Injury, Therapy and Rehabilitation. Blackwell Science Scientific Publications Limited, Oxford.

Bromiley, M (1989) Cashes Textbook for Physiotherapists: Veterinary Physiotherapy. Faber and Faber Ltd. Great Britain.

Calder, J (1970) The Dog as a Patient. Physiotherapy, 56, 2, 60-61.

Hopes, R (1970) Physiotherapy in the Horse. Physiotherapy, 56, 2, 56-59

Knowless, D, Mackintosh, S (1994) A survey of Animal Physiotherapy Practice in Britain. Physiotherapy, 80, 5, 285-288.

Lawson, D (1970) Physiotherapy in Veterinary Medicine. Physiotherapy, 56, 2, 53-55.

Mackintosh, S, McNamara, K (1993) Veterinary Surgeons' Perceptions of Animal Physiotherapy. Physiotherapy, 79, 5, 312-316.

Mills, DL, Levine, D (1997) The Role of Exercise and Physical Modalities in the Treatment of Osteoarthritis. Veterinary Clinics of North America: Small Animal Practice, 27, 4, 913-929.

Saywell, S (1970) Physiotherapy in Veterinary Work: An Introduction. Physiotherapy, 56, 2, 52-53.

Steiss, JE (2002) Muscles Disorders and Rehabilitation in Canine Athletes. Veterinary Clinics of North America: Small Animal Practice, 32, 1, 267-285

Stewart, JL (2002) Kissing Spines in Horses. PhysioForum, 16, 7, 12-14.

Taylor, GA (1970). Treating Animals with Short Wave Diathermy. Physiotherapy, 56, 2, 62-64.

Thompson, N "Veterinary Acupuncture", http:// www.natural-animal-health.uk.au.

Walt, AM, Jeune, RM, Stewart, JL (2002). Muscular Pain as a Result of Forelimb Lameness in the Horse. PhysioForum, 16, 4, 4-5.

Wihelm,S (2003) South African Association of Physiotherapists in Animal Therapy. Physioforum, 17, 2, 12-13 